\title{
The detection of crackles based on mathematical morphology in spectrogram analysis
}

\author{
Kexin Zhang ${ }^{\mathrm{a}, \mathrm{b}}$, Xuefeng Wang ${ }^{\mathrm{c}}$, Fangfang $\mathrm{Han}^{\mathrm{a}}$ and Hong Zhao ${ }^{\mathrm{a}, *}$ \\ ${ }^{a}$ Northeastern University, Shenyang, Liaoning, China \\ ${ }^{\mathrm{b}}$ Liaoning University of Traditional Chinese Medicine, Shenyang, Liaoning, China \\ ${ }^{\mathrm{c}}$ The First Affiliated Hospital of Liaoning University of Traditional Chinese Medicine, Shenyang, \\ Liaoning, China
}

\begin{abstract}
.
BACKGROUND: Crackles are very common abnormal breath sounds in the lung and can be used to diagnose pulmonary diseases.

OBJECTIVE: In this study, a method is proposed for the detection of adventitious transient sounds from normal breath sounds. METHODS: This method automatically recognizes crackles based on the extraction and analysis of spectral information from digitally recorded lung sounds. Various mathematical morphology feature sets were extracted through wavelet spectrogram analysis on pulmonary signals. In order to evaluate the effects of different wavelets types on crackle detection, different wavelets were tested.

RESULTS: The results showed that the proposed method achieved an $86 \%$ accuracy in the detection of crackles.

CONCLUSIONS: The spectrograms of the crackles in the lung exhibit irregular ellipse image features. For lung sound analysis,
\end{abstract} this is a useful feature that can be used for the immediate recognition and analysis of crackles.

Keywords: Crackles, mathematical morphology, wavelets, lung sounds, spectrogram

\section{Introduction}

Crackles in the lung are discontinuous, adventitious non-musical respiratory sounds that are attributed to sudden bursts of air within bronchioles. They often accompany respiratory diseases. Morphologically, crackles are explosive and transient. The inherent properties of pulmonary crackles such as timing, epochs of occurrence, and pitch can be used in the diagnosis of a variety of pulmonary diseases such as pneumonia, bronchiectasis, fibrosing alveolitis and asbestosis [1].

Crackles are frequently observed in patients with cardiorespiratory diseases. The duration of a crackle is less than $20 \mathrm{~ms}$, and the frequency content is typically wide, ranging from 100 to $2000 \mathrm{~Hz}$ or even higher [2].

\footnotetext{
${ }^{*}$ Corresponding author: Hong Zhao, 308 Admin Building, Neusoft Park, \#2 Xinxiu st. Hunnan dist. Shenyang, Liaoning 110179, China. Tel.: +86 0248366 1111; E-mail: zhaoh@ neusoft.com.
}

0928-7329/15/\$35.00 (c) 2015 - IOS Press and the authors. All rights reserved

This article is published online with Open Access and distributed under the terms of the Creative Commons Attribution NonCommercial License. 

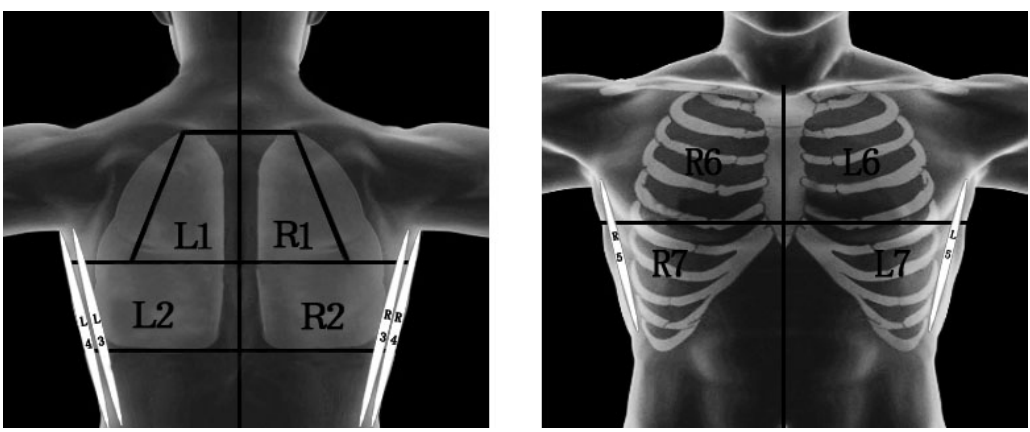

Fig. 1. Acoustic signal recording positions.

In present researches, commonly used signal processing techniques are fast Fourier transform (FFT), autoregressive model (AR), fractal-dimension (FD) analysis, mel frequency cepstrum coefficients (MFCC), and wavelet analysis. Most researchers adopt mathematical techniques to extract the features of crackle signals. Signal processing methods are usually used only in time or frequency domain, and few researchers managed to analyze the lung sound signals in time-frequency domain [3-7]. In this study, the short-time Fourier transform (STFT) spectrograms of crackles were analyzed and a new visual feature of crackles was extracted. A mathematical morphology algorithm was designed for the automatic recognition of crackles.

The remainder of this paper is organized as follows. Section 2 describes the data used in this study. Section 3.1 puts forward a new visual feature of crackle based on its spectrogram; Section 3.2 describes the method of spectrogram bitmap conversion; Section 3.3 describes the mathematical morphology algorithm for crackle automatic recognition. Finally, Section 4 concludes this study and Section 5 presents a discussion.

\section{Experimental data}

The dataset used in the present study consists of 100 breath sound signals, including 50 crackles and 50 normal sounds. The clinical diagnosis classification of those signals was performed by two physicians.

The acoustic signals were recorded using an electronic stethoscope (Model 4000, 3M, Inc., USA) from several positions of the body. The positions are illustrated in Fig. 1, where L1 indicates left record region 1 and R1 indicates right record region 1, and so on. The stethoscope can collect six separate sounds, as digital data, to playback at normal or half speed-to aid in the diagnosis. Each sound file included $8 \mathrm{~s}$ of recording at a sampling rate of 8000 samples per second, using 16 bits acoustic signals. All the wave files had been converted to .wav format in advance for the following analysis. All recording procedures took place at the First Affiliated Hospital of Liaoning University of Traditional Chinese Medicine (LNUTCM), Shenyang, China.

\section{Methods}

\subsection{Spectrogram}

A spectrogram can represent the dynamics of spectral density through a time-varying spectral representation [8]. Spectrograms were extracted from the time signals using STFT in this study. The spectrogram of a signal $s(t)$ can be estimated by computing the squared magnitude of the signal's STFT, as 

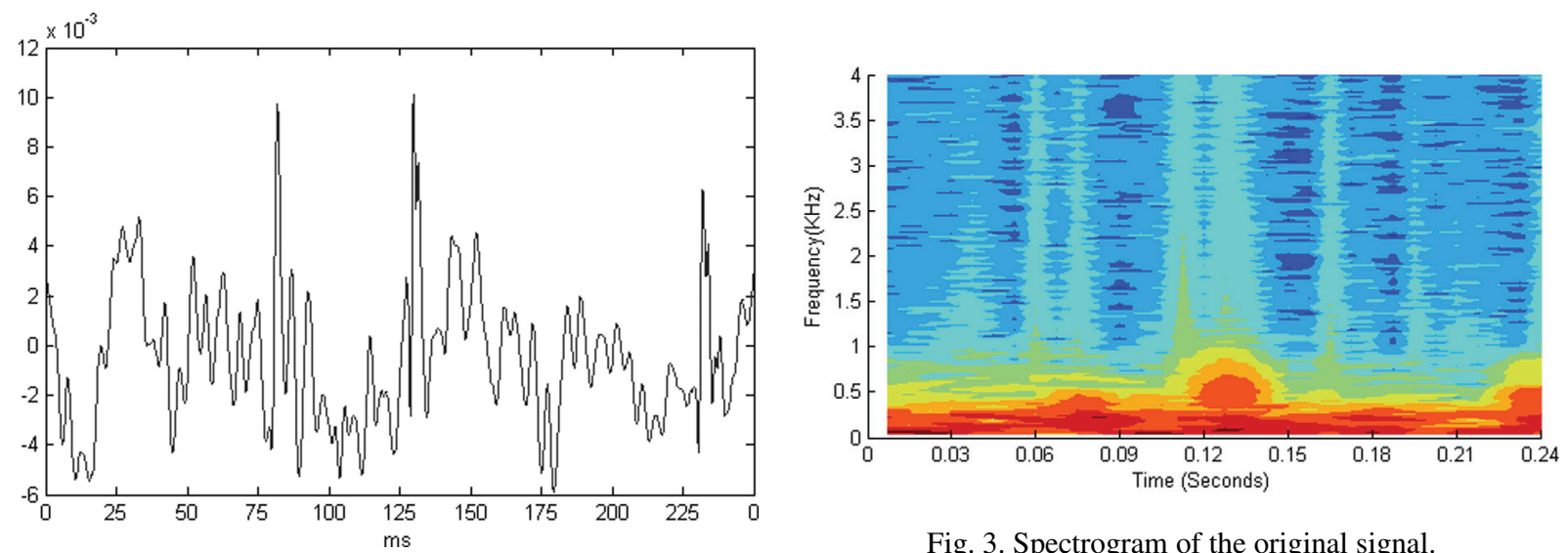

Fig. 3. Spectrogram of the original signal.

Fig. 2. The 250 ms waveform of original sound (2000 points).

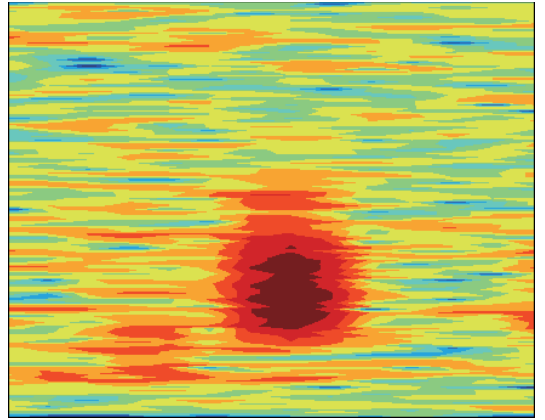

(i)

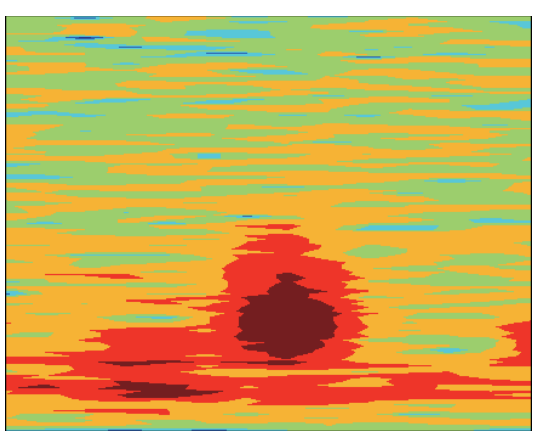

(ii)

Fig. 4. Spectrograms of wavelet for an original signal. (i) Spectrogram obtained by Db3 wavelet decomposition (ii) Spectrogram obtained by Bio22 wavelet.

Eq. (1) below shows:

$$
\operatorname{Spectrogram}(t, \omega)=|\operatorname{STFT}(t, \omega)|^{2} \text {. }
$$

Figure 2 shows the original $250 \mathrm{~ms}$ waveform of a crackle, and its STFT spectrogram is shown in Fig. 3. The spectrogram was obtained using the Matlab program and saved to a pseudo-color bitmap in the solution of $435 \times 343$ pixels.

Almost every crackle signal's spectrogram includes at least one red eclipse area, therefore it might be a feature of crackle spectrograms. In order to study the images more thoroughly, the Wavelet Decomposition [9]10] of signals were calculated. Multilevel 1-D wavelet decomposition performs multilevel one-dimensional wavelet analysis with a specific wavelet.

In order to analyze the crackle signals, 16 kinds of 1-D Wavelet bases were adopted, some of which ( db2, db3, bior2.2 and bior3.1) were proved more suitable for the eclipse symbol recognition than the others in this study. Spectrograms of wavelet for an original signal is shown in Fig. 4.

\subsection{Bitmap conversion}

The pseudo-color bitmap was converted to a grey bitmap first and then histogram equalization was performed to enhance the high gray area. Assuming a discrete grayscale image $\{x\}$ and let $n_{i}$ be the 


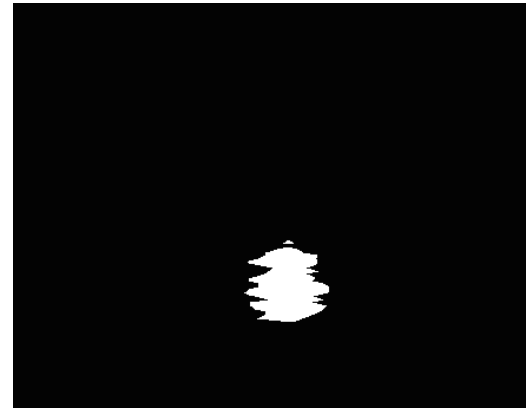

(i)

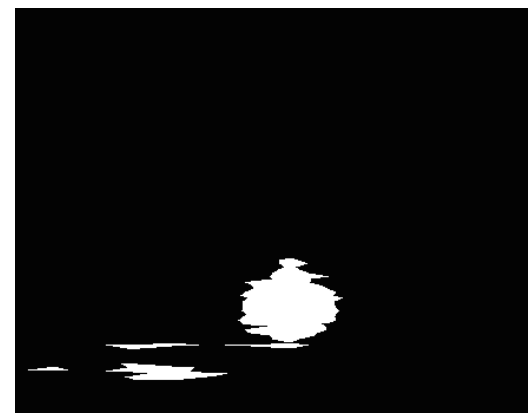

(ii)

Fig. 5. Spectrograms converted to black-and-white 2-bit bitmaps. (i) Db3 wavelet black-and-white Spectrogram (ii) Bio22 wavelet black-and-white Spectrogram.

number of occurrences of gray level $i$, the probability of the occurrence of a pixel of level $i$ in the image is

$$
p_{x}(i)=p(x=i)=\frac{n_{i}}{n}, \quad 0 \leqslant i<L,
$$

where $L$ is the total number of gray levels in the image, $n$ is the total number of pixels in the image, and $p_{x}(i)$ is the image's histogram for the pixel value $i$ in fact, normalized to $[0,1]$.

Then, the cumulative distribution function corresponding to $p_{x}$ could be defined as

$$
c d f_{x}(i)=\sum_{j=0}^{i} p_{x}(j)
$$

Formula (3) also describes the image's accumulated normalized histogram.

The grey bitmap spectrogram was converted to a black-and-white 2-bit bitmap by setting the threshold value to 0.984 or 0.875 . Afterward, the ellipse areas became the main objects in the spectrogram. Figure 5 presents some sample black-and-white spectrograms.

\subsection{Mathematical morphology}

Mathematical morphology (MM) is a theory as well as a technique for the analysis and processing of geometrical structures [11]. MM has also gained popularity in digital image processing because it could be applied to graphs, surface meshes, solids, and many other spatial structures.

The following parameters were used as the Spectrogram properties:

(1) Max area

(2) Centroid

(3) Major axis length

(4) Minor axis length

(5) Eccentricity

In mathematics, eccentricity, denoted by $e$, is a parameter associated with every conic section. It can be treated as a measure of how much the conic section deviates from being circular.

(6) Euler number

Euler number is a scalar that specifies the number of objects in the region minus the number of holes in those objects. 


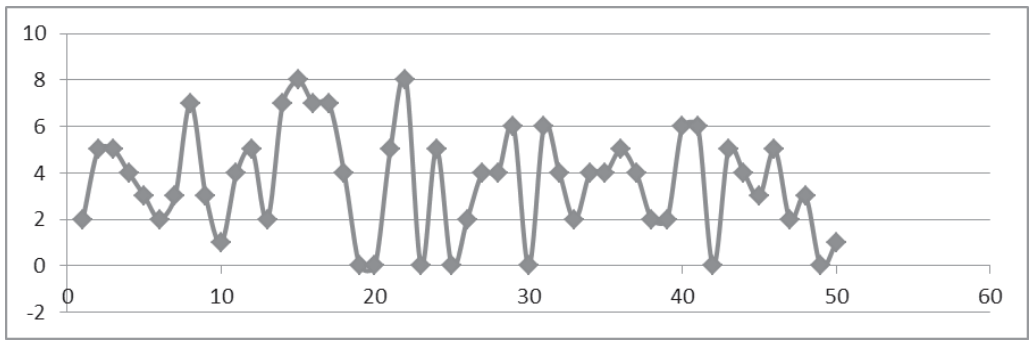

Fig. 6. Recognition of 50 crackles (crackles' $y$ values were great than 0 ).

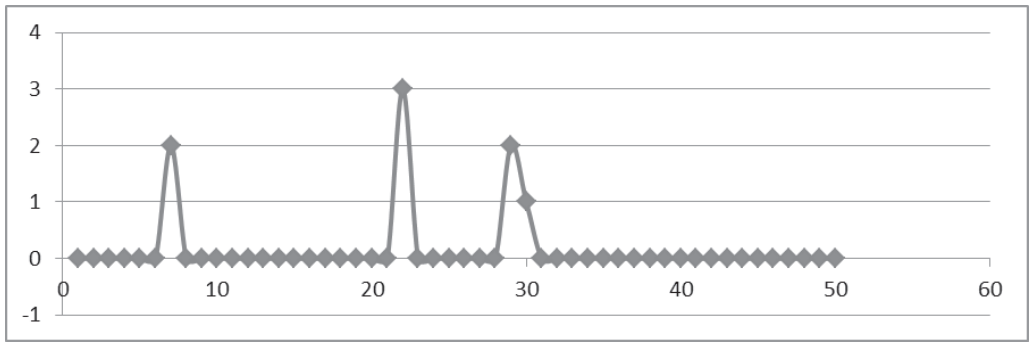

Fig. 7. Recognition of 50 normal breath sounds (normal breath sounds' $y$ value should be 0 ).

The eigenvalue of the crackle is

$$
y(\text { crackle })=\sum_{i=1}^{n} a_{i} p_{i}
$$

$n$ indicates 9 kinds of transforms; $p_{i}$ is a numerical value of $(1,0,-1)$, which express the crackle, the normal breath and other lung sound; $a_{i}$ is the weighted value of the wavelet, and the initial value is $1 . p_{i}$ is obtained by the mathematical morphology calculation and its parameter's optimum value is determined by feedback learning.

\section{Results}

With all respiratory sounds evaluated separately and no relation established between the lung sound and the patient, the algorithm presented the results shown in Fig. 6. Positive values were computed when the technique returned a positive value containing crackle, while negative values were computed when the technique returned a negative value without crackles. A lung sound was considered as crackle if its $y$ (crackle) value was great than 0 . The automatic recognition method was validated satisfying clinical criteria. With this method, $86 \%$ crackles were detected correctly, while $8 \%$ normal breath sounds were incorrectly recognized as crackles (Fig. 7).

\section{Conclusion and discussion}

The spectrograms of the crackles in the lung exhibit irregular ellipse image features. For lung sound analysis, this is a useful feature that can be used for the immediate recognition and analysis of crackles. 
This study demonstrated that Mathematical Morphology in Spectrogram Analysis is an effective method to detect crackles. Morphology Parameters, such as Max Area, Centroid, Major Axis Length, Minor Axis Length, Eccentricity and Euler Number can be used in crackle recognition.

The linear features contained by part of the crackles may reduce the crackles' feature scores. In addition, some isolated crackles are not significant, making them difficult to be determined. Therefore, it is difficult to fully confirm the diagnosis. However, the numerical analysis method proposed in this paper provides clinicians reasonable tips with visualization.

\section{Acknowledgement}

This work was supported by the National Natural Science Foundation of China(81273800).

\section{References}

[1] Serbes, G., et al., Pulmonary crackle detection using time-frequency and time-scale analysis, Digital Signal Processing (2013)23(3): 1012-1021.

[2] Parkhi, A. and M. Pawar, Analysis of deformities in lung using short time fourier transform spectrogram analysis on lung sound, Computational Intelligence and Communication Networks (CICN), 2011 International Conference.

[3] Palaniappan, R., et al., CompuTER-based respiratory sound analysis: A systematic review, Iete Technical Review (2013)30(3): 248-256.

[4] Zhenzhen, L., et al., A novel method for feature extraction of crackles in lung sound, Biomedical Engineering and Informatics (BMEI), 2012 5th International Conference.

[5] Zhenzhen, L. and D. Minghui, HHT based lung sound crackle detection and classification, Intelligent Signal Processing and Communication Systems, 2005. ISPACS 2005. Proceedings of 2005 International Symposium.

[6] Hadjileontiadis, L. J. and I. T. Rekanos, Detection of explosive lung and bowel sounds by means of fractal dimension, Signal Processing Letters, IEEE (2003)10(10): 311-314.

[7] Hadjileontiadis, L. J., A texture-based classification of crackles and squawks using lacunarity, IEEE Trans Biomed Eng (2009)56(3): 718-732.

[8] Boashash, B., editor, Time-frequency signal analysis and processing - a comprehensive reference, Elsevier Science, Oxford, 2003

[9] Ayari, F., et al., Wavelets: An efficient tool for lung sounds analysis, Computer Systems and Applications, 2008. AICCSA 2008. IEEE/ACS International Conference.

[10] Hu, S., et al., Texture feature extraction based on wavelet transform and gray-level co-occurrence matrices applied to osteosarcoma diagnosis, Bio-Medical Materials and Engineering (2014)24: 129-143.

[11] Zhang, E., et al., Automatic detection of microcalcifications using mathematical morphology and a support vector machine, Bio-Medical Materials and Engineering (2014)24: 53-59. 